\title{
Cytoskeletal Mechanics
}

\author{
Vaishnavi Ananthanarayanan*
}

The cytoskeleton, as its name suggests, is a class of self-assembling polymers that dictate the shape and mechanical properties of cells. Comprising the actin filaments, microtubules, and intermediate filaments, the cytoskeleton also teams up with motor proteins to determine the relative positions of membrane-bound compartments within cells and bring about other vital cellular functions, including migration and cell division. The dynamicity of cytoskeletal polymers also sets them apart from their namesake in our bodies (the skeleton) by allowing them to adapt to external and internal stimuli quickly. For instance, when presented with a bacterium, the immune system's phagocytic cells form actin protrusions on-demand to engulf the invaders. Wound healing requires the concerted rearrangement of both the actin and microtubule cytoskeleton to enable cells to migrate to the site of the wound. Differentiation of stem cells can be controlled by tweaking the organization and abundance of the actin and microtubule cytoskeleton when altering the cellular substrate. Therefore, in the case of the cytoskeleton, 'form follows function,' but also 'function follows form'! All these examples underscore the role of the cytoskeleton in normal cellular functions.

In this edition of the Journal of the Indian Institute of Science, reviews by Hegde, A. et al., Biswas, A. et al., Rasool, S. et al., and Kumari, S. et al. delve into the essential roles of the cytoskeleton and associated proteins in wound healing, membrane homeostasis, cell-cell interactions, and in the formation of the immunological synapse, respectively. Paradkar, S. et al. and Yadu, N. et al. describe unconventional cytoskeletal assemblies in red blood cells and bacteria, which help the former deform, and the latter divide. Krishnamurthy, V. dons an active matter lens in his article to describe the actomyosin cortex underlying the cell membrane.

The cytoskeleton also proves to be an important target during disease, when normal cellular processes are derailed. Cancer cells typically display lower mechanical stiffness when compared to healthy counterparts. Softer cancer cells are proposed to be able to squeeze through narrow blood vessels to spread to regions far from the original cancer site, thereby aiding metastasis. Viruses highjack motor-driven transport on the microtubules to reach their replication sites, leading to increased infectivity.

The centrosome is the microtubule-organizing centre in most animal cells. Here, Jaiswal et al. elucidate how the centrosome is assembled and discuss how centrosomal abnormalities give rise to human diseases. Pore-forming toxins (PFTs) are bacterial proteins that bind and form pores on animal cell membranes to increase infection severity. In their review, Kumar, H. et al. delineate the cytoskeleton's multiple roles in responding to PFTs, with some of these beneficial to the host cell and others advantageous to the bacterium. Finally, Jain, K. et al. review efforts in the motor protein field to understand how multiple motor proteins work together to effect intracellular transport.

As is evinced from a reading of these excellent reviews, an informed description of the cytoskeleton and associated proteins' form and function requires the close collaboration of scientists in diverse fields, including but not limited to Biology, Physics, Mathematics, and Engineering. With the articles in this special edition, I hope the reader gets the flavor of the possibilities as well as roadblocks in future research in the area. I thank the Editor-in-Chief, Prof. G. K. Ananthasuresh for the opportunity to serve as Editor of this special issue, and the staff of the Journal of the Indian Institute of Science, and Ms. Kavita Harish and Ms. Leelakshi from the Office of Communications at IISc for their assistance in publishing this special edition.

\section{Publisher's Note}

Springer Nature remains neutral with regard to jurisdictional claims in published maps and institutional affiliations.

Accepted: 14 December 2020

Published online: 7 January 2021
${ }^{1}$ EMBL Australia Node in Single Molecule Science, School of Medical Sciences, University of New South Wales, Sydney, Australia. *vaish@unsw.edu.au 